\title{
Research on Staff Explicitation in Organizational Knowledge Management Based on Fuzzy Set Similarity to Ideal Solution
}

\author{
Zhang Qipeng ${ }^{1, *}$, Tong Biao ${ }^{2}$, Li Liang ${ }^{3}$, Zhang Qiuxue ${ }^{4}$ and Zhou Dandan ${ }^{2}$
}

${ }^{1}$ Department of Business Administration, Zhejiang University of Technology, Zhejiang Hangzhou 310023, China;
${ }^{2}$ Department of Technical Economics, Zhejiang University of Technology, Zhejiang Hangzhou 310023, China;
${ }^{3}$ Department of Finance, Zhejiang University of Technology, Zhejiang Hangzhou 310023, China; ${ }^{4}$ Department of Intel-
lectual Property Rights, Zhejiang University of Technology, Zhejiang Hangzhou 310023, China

\begin{abstract}
In this paper, we prompt that the promoting relationship between three layers of DKMC model based on the fuzzy set similarity to ideal solution. The knowledge fundamental competence impulses the knowledge transfer competence, and the knowledge transfer competence impulses the knowledge dynamic application competence. It is also proved that the knowledge dynamic application competence, which pushes the OP by organization innovation, is the key of the DKMC model to shift the OP. It brings forward the evaluation system of the DKMC. At the first time, it utilizes particle swarm optimization method in the field of the colony intelligence into the optimization of the evaluation model, so as to recommend a full set of methods to optimize DKMC evaluation model based on the fuzzy set similarity to ideal solution.
\end{abstract}

Keywords: Algorithm, fuzzy set similarity to ideal solution, organizational knowledge management, the staff explicitation.

\section{INTRODUCTION}

Recently, the researcher has witnessed many organizations are taking cognizance of and implementing knowledge management $(\mathrm{KM})$ in management practice. $\mathrm{KM}$ is the management activities of knowledge's producing, processing, broadcasting and applying in order to keep or sharpen the competitive edge. KM also has a close relationship with the changeful competition circumstance, so it is necessary for the organization to be dynamic as well. The KM objective targets are not only for integration of the internal and the external knowledge [1,2], but also for the development of organization performance (OP).

The dissertation analyses internal foundation of organization dynamic competence from the view of KM, and demonstrates dynamic knowledge management competence (DKMC) with its triple-layered structure model [3]. The model consists of foundation layer which lies in KM fundamental competence, conversion layer which lies in $\mathrm{KNi}$ transfer competence and application layer which lies in KM dynamic application competence. It points out the repository which contains visible and invisible knowledge stored in each one's head of the organization is the core of the DKMC triple- layered structure. On the one hand, the capability of each layer accumulates to enlarge the knowledge repository. On the other hand, the knowledge repository has a great effect on each layer. Moreover, different emphasizes are laid on the relationship of each layer in the knowledge repository.
Otherwise, DKMC model focuses on dynamic configuration to suit the changing environment. The model also shows the status and effect of each layer so as to target the KM level for organizing itself and to identify the problems on corresponding layer [4]. It helps to set up a holistic KM solution for the competence and resource adapting to dynamic circumstance $[5,6]$, while the consisting competing advantage can be obtained.

Knowledge management is the product of the era of knowledge economy, and the development of knowledgebased economy depends on the effective management of knowledge: From a macro point of view, knowledge management is an important driving force for the development of knowledge-based economy; while from the microscopic point of view, knowledge management through a series of core functions effectively optimize the enterprise's business activity. And knowledge management is not only a strategy and ideas, but also a workable technology. While at the same time, the history of the rise and fall of the knowledge management calls for a holistic and targeted knowledge management is done. Knowledge management should first be based on the level of corporate strategy, rather than a restricted matter, thus it must be implemented as a whole. Knowledge management doesn't cover everything, but should be focused on the basis of actual business in order to achieve optimal performance. Management knowledge life cycle has become the new ideas of modern knowledge management [7].

Today, the majority of executives are aware of the strategic importance of knowledge. Moreover as in other areas of management, we do not lack a useful framework, alternative models and checklists directory to be chosen from. However, 
it seems that some solutions, just like Band-Aid, can be used under any circumstances. When a lot of knowledge managers selected error framework one after another, the idea that knowledge management is the process of the evolution of the knowledge life cycle will have a profound impact.

An important function of knowledge management is the management of the whole knowledge life cycle. How to manage the knowledge in a complex ecology of knowledge, it requires us to abandon the peculiar and mechanical reductionism that means the whole is to be divided into individual to understand and explain, instead pick up the weapons of complexity theory, using such a system life cycle thinking to take care of the knowledge mufti-attribute, muftidimensional value and a variety of factors. In the book" White Paper on Knowledge Management: Secondgeneration knowledge management", knowledge life cycle that appears repeatedly is based on organizational learning theory and complexity theory, which are the basis of the second generation of knowledge management concepts.

\section{THE BACKGROUND AND FRAME WORK OF ORGANIZATIONAL KNOWLEDGE MANAGEMENT IN CHINA}

The implementation of knowledge management in the enterprises in China has not achieved the desired effect. The successful experience is very few and failure's is more. The other hand, many Western companies, knowledge management project is a mufti failed. The reasons for the failure are many and varied, but we attempt to analyze the two important reasons:

(1) The lack of a deep understanding of enterprise knowledge management, it thinks only knowledge as the object of study of knowledge management, while ignoring the important role who is closely related to the environment (in particular, the operation of the internal environment). Many knowledge management projects are simply seen as an extension of information management, while too much emphasis on the technical attributes of knowledge management, and neglect the social attributes of knowledge management.

(2) Enterprise in importing knowledge management, due to the lack of clear understanding on their own level of knowledge management capability status quo, so it is unclear whether the organization has ready. After the implementation of knowledge management, there is no a clear effect evaluation mechanisms and standards, so the employees feel that knowledge management can not bring real benefits to the enterprise [8]. These will make the project a mere formality and failure ultimately.

The existing researches on knowledge management maturity model widespread a problem: Model construction methods are all experienced judgment and lack of real data on the selection of elements. Therefore, this study attempts to use experience research (rather than empirical judgments) to determine the composition of the enterprise knowledge management maturity model dimensions. Throughout the course of the study, we found that:

(1) There is a new method based on empirical research to Knowledge Management Maturity Model.
(2) The effectiveness of knowledge management maturity model constructed in this study based on the operation of the environmental perspective is good. The model has been adopted in DHI"DCW, and one of the reference based on the model has to develop as a human resources management system.

(3) Whether or not knowledge management project has been implemented, there is knowledge management operation environment objective in enterprises, and this environment can be measured. From the point of view of knowledge management operation environmental factors, enterprise can clear the level of maturity of knowledge management through the evaluation of the operation environment construction, and thus appropriate to grasp the import of the timing and focus of knowledge management direction.

(4) Overall, knowledge management operation environment will have an impact on business performance through the intermediary of knowledge management core processes. The detailed impact relationships are: Centralization of enterprise knowledge management operation environment has a negative impact on knowledge management acquisition process and knowledge-sharing processes in knowledge management core processes, and formal environmental has a negative impact on knowledge-sharing processes and knowledge innovation process. Technical support for knowledge processes has a positive impact on the processes of knowledge acquisition and knowledge sharing. Bureaucratic culture has a negative effect on knowledge innovation, and innovative culture has positive effect on knowledge acquisition, knowledge innovation, and the same time, the supportive culture has positive effect on knowledge sharing and knowledge innovation. The core Knowledge processes (knowledge acquisition, sharing, and innovation) has a positive effect on Enterprise Performance.

(5) Codified-knowledge management strategy play a positive regulatory role on the relationship of knowledge acquisition, knowledge sharing and firm performance, but play a negative regulatory role on the relationship of knowledge innovation and firm performance.

(6) The interaction of value of human resources strategy, knowledge sharing and knowledge innovation has a positive impact on business performance. The effect of the interaction of the value of human resources strategy and knowledge acquisition on firm performance is not significant. The interaction of the specificity of human resource and knowledge innovation has a positive impact on enterprise performance.

(7) The conclusions of relationship between knowledge management operation environment the knowledge management core process and enterprise performance can be applied to the construction of "a knowledge management maturity model".

\section{ORGANIZATIONAL CULTURE DIMENSIONS AND KNOWLEDGE MANAGEMENT SUCCESS}

First, persistent learning culture is productive to improving an enterprise's efficiency in knowledge obtaining and accumulation, whereby to promote KMS. As well as accumulating and acquiring knowledge from inside whenever and wherever, firms equipped with high learning aptitude 
and motives, are more adept in from rivals and coalition partners gaining access to new knowledge, which is universally acknowledged as a way to success as no firm can thoroughly have the ownership of or self develop all knowledge for achieving competitive advantage. So, persistent learningtyped OC can stimulate a motive for staff to create, own, and absorb new knowledge, and endow KM with a more copious connotation, thus effectively extending the platform of it.

Next, continuity of learning-typed culture is a driver of scientific originality, furnishing KM with the soul and momentum. There are two basic prerequisites for individuals to effect a highly efficient innovation of knowledge: reasonable knowledge structure with both solid specialist and encyclopedical related knowledge and scientific thinking methodology. To obtain such a knowledge constitution leans on permanent study. Further, innovative thoughts methods are a key to the scientific novelty course as only if innovators must bring into full play thinking functions: boldly, diligently, artfully and profoundly deliberate, efficient innovation would be fulfilled. The availability of the methodologies is subject to in-depth pursuit of theory and sustainable learning-by-doing.

Third, incessantly learning environment makes for raising the knowledge-sharing efficiency to push knowledge administration to move toward the bank of victory. High efficiency in knowledge sharing, marking a successful KM, is determined by three fundamental factors: intentions and capability of senders and recipients, and knowledge's own tacit attribute. Knowledge dispatchers must not only guarantee others to share knowledge but also be capable of employing a manner of ease of understanding to remit the knowledge they have already mastered, particularly the ambiguous one, which requires continuous study.

In the case of receivers, only if they must have a high spirit of permanent reading, then they can possess a strong motive for accepting and taking in the knowledge coming from senders. Eventually, constant learning-typed culture contributes to advancing the efficiency in knowledge diffusion and usage. The knowledge innovatively created during the course of business operations or obscured from coalition partners, provided to be appropriately disposed and developed the full use of, can coin adequate value. Only in the cultural study atmosphere where staff is in the habit of taking in fresh knowledge, proper allocation of knowledge can be achieved, prompted by discriminative employees. This is in favor of transforming of personnel from "being asked to disseminate and employ knowledge to "being ready to" and facilitates them to be keen on unceasing study of how to maximize the efficacy of a new skill, ultimately upgrading the value creativity for running knowledge.

At organizational level, firms should try to translate each individual's intangible knowledge into tangible and systematic knowledge, and to promote interactions, both formal and informal, among individuals and groups. At individual level, a person must know how to hold skills necessary for work, obtain access to know-how from colleagues, share working experience and techniques with others. This, of course, can lead to a high probability of KMS. At a start, appropriate power distance contributes to an unobstructed channel for information communication and the reduction of information ullage. Spreading knowledge at a high efficiency requires correct identification of information and precise distribution for specific staff, thus accurate recognization concerning knowledge requesting and swift processing are a vital chain for over the course of disseminating knowledge. However, at businesses with a long distance of authority, the management bureaucracy level is in the habit of supervising personnel through the formal commanding system at each tier and information communication follows the route from the upper level to the lower level and then from the lower level to the upper level till to the chief executive. On this track, severe wearing and discounting of timely delivery in connection with data provokes missing chance or suspension of handling of knowledge request. Notably, lengthy power-distance enterprises fail to accomplish a high efficient circulation of information and suffer a low efficiency of spreading out.

Next, suitable authority space is a contributor of upgrading employees' work autonomy, insuring diversity and smoothing of information-enjoying channels. Low powerspace firms underscore empowerment-styled management, which means democratic involvement and supporting staff to make up a volunteer team for obligatory performing and is embodied in improving staff's self-decision power, giving strong backing to employees in having access to various working resources like technique to guarantee them to independently carry out significant undertakings, and motivating them to take on greater responsibilities via job shift and enrichment. While, long distance of power may induce the knowledgeable members of staff to be inclined to treat knowledge as a source of expert power for the sake of snatching more bargaining capitals to upgrade their own status and obscure economic interests. Under the circumstance of modest power expanse, however, they may have an impulsion for going shares of their intellectual results.

Third, appropriate power distance is a driver of negotiation-based or constructive communication modes, which makes sure the pattern of power exercise based on guidance and security functions, so as to advance knowledge innovation ability to prompt successfully running knowledge. Uniqueness concerning knowledge per se makes its novelty be achieved just under the circumstance of high autonomy of interest-related persons, over the course of which power application is positioned in the serving form. This form is in contrast with the counterpart in enterprises with a long power space, authoritative and manipulative. Evidently, the former style is more positive than the latter in knowledge innovation.

\section{THE ALGORITHM OF FUZZY SET SIMILARITY TO IDEAL SOLUTION}

Technique for Order Preference by Similarity to Ideal Solution (SIS) method is also one of those useful multicriteria decision making method for surveying issues in real world for the first time. This method was also suggested by Jahanshahlo et al. They described it as:

Let $A_{1}, A_{2}, \ldots, A_{m}$ be $m$ alternatives, which are supposed to be ranked by $k$ decision makers based on $n$ criteria $\left(C_{1}, C_{2}, \ldots, C_{n}\right)$. Let $X_{i j}$ be the rating score of $A_{i}$ associ- 
ated with $j^{\text {th }}$ criteria and is defined as $x_{i j} \in\left[x_{i j}^{l}, x_{i j}^{u}\right]$. Weights of criteria are defined as $w_{1}, w_{2}, \ldots, w_{n}$, where $w_{j}$ is the weight of $C_{j}$. We can define an MADM problem with interval numbers briefly in a decision making matrix.

In SIS method with interval numbers we have to normalize decision making matrix as we show it below:

$$
\begin{aligned}
& \tilde{a}_{i j}^{l}=\frac{x_{i j}^{l}}{\sqrt{\sum_{j=1}^{m}\left[\left(x_{i j}^{l}\right)^{2}+\left(x_{i j}^{u}\right)^{2}\right]}}, i=1,2, \ldots, n, j=1,2, \ldots, m \\
& \tilde{a}_{i j}^{u}=\frac{x_{i j}^{u}}{\sqrt{\sum_{j=1}^{m}\left[\left(x_{i j}^{l}\right)^{2}+\left(x_{i j}^{u}\right)^{2}\right]}}, i=1,2, \ldots, n, j=1,2, \ldots, m
\end{aligned}
$$

Now $\left[x_{i j}^{l}, x_{i j}^{u}\right]$ are normalized and the calculated domain $\left[a_{i j}^{l}, a_{i j}^{u}\right]$ belongs to $[0,1]$. Because of the differences in importance of each criterion, in the next step we will calculate weighted normalized decision matrix with interval numbers as below:

$\tilde{v}_{i j}^{l}=w_{j} \tilde{a}_{i j}^{l}, i=1,2, \ldots, n, j=1,2, \ldots, m$

$\tilde{v}_{i j}^{u}=w_{j} \tilde{a}_{i j}^{u}, i=1,2, \ldots, n, j=1,2, \ldots, m$

where $w_{i}$ is the weight of the $i^{t h}$ criterion and $\sum w_{i}=1$.

Fuzzy set is an extended form of classic set introduced by Zadeh. In a classic set, each element has two values. In other words, an element either belongs to a set or not. If an element becomes a member of set $\mathrm{A}$, its related value is equal to 1 , and zero, otherwise. However, fuzzy theory is attributing a number between $[0,1]$ to each $x$ from $X$.

A Convex Fuzzy Set: The "A" fuzzy set is convex if and only if for each $x_{1}, x_{2} \in X$ and each $\lambda \in[0,1]$, we have

$\mu_{A}\left[\lambda x_{1}+(1-\lambda) x_{2}\right] \geq \min \left[\mu_{A}\left(x_{1}\right), \mu_{A}\left(x_{2}\right)\right]$

For real-world applications, we utilize some fuzzy and vague statements rather than some crisp terms. Very low, low, middle, high and very high are some examples of linguistic terms. Fuzzy numbers can stand for these linguistic terms in a mathematical model. In this paper, the importance weights of the ratings of qualitative criteria are considered as linguistic variables. In this paper, the decision-makers use the linguistic variables shown in Table 1 to evaluate the ratings of alternatives with respect to qualitative criteria.

In this article, we select a supplier that has the low amount of risk in relationship with the company. This problem can be defined as a group MADM (GMADM) problem. We consider some steps for modeling these cases as below:

a) $\mathrm{A}$ set of $\mathrm{k}$ decision maker that is defined by $D=\left\{D_{1}, D_{2}, \ldots, D_{k}\right\}$;

b) A set of $\mathrm{m}$ supplier (alternative) that we call it $A=\left\{A_{1}, A_{2}, \ldots, A_{m}\right\}$;

c) A set of $\mathrm{n}$ criteria that we evaluate suppliers by those criteria and call it $C=\left\{C_{1}, C_{2}, \ldots, C_{n}\right\}$;

d) A set of performance rate of supplier, $A_{i}(i=1,2, \ldots, m)$ in association with criteria $\left(C_{j}, j=1,2, \ldots, n\right)$ that we show it as $X=\left\{x_{i j}, i=1,2, \ldots, m, j=1,2, \ldots, n\right\}$.

Consider a situation where there are $k$ decision makers in a group and each decision maker $D_{k}$ and $k=1,2, \ldots, k$ evaluates suppliers based on fuzzy numbers $\mu_{\tilde{A}}(x)$ that has a membership degree $\mu_{\tilde{A}}(x)$. After choosing adequate linguistic terms for ranking suppliers and translating them to fuzzy numbers, we utilized $\alpha$-cut method to alternate these fuzzy numbers by interval numbers instead of defuzzification for prioritizing alternatives and then we used a SIS method to rank interval numbers for ranking suppliers.

\section{THE EXPERIMENT AND DATA ANALYSIS}

Samples are chose for the questionnaire test. The experiment data of sensitivity of KM of the sample is shown in Table 1. The experiment data of stable of KM of the sample is shown in Table 2.

Knowledge management and learning organization is the product of the era of knowledge economic society. They have an inextricably close relationship. The learning organization is a carrier of the implementation of knowledge management, and knowledge management is the core of learning organization.

Fig. (1) shows the volatility of the sample. The prior research theoretically provides salutary lessons and guidance

Table 1. Sensitivity of KM.

\begin{tabular}{|c|c|c|c|}
\hline \multirow{2}{*}{ Cipher text image } & \multicolumn{3}{|c|}{ Fine Tuning Key } \\
\cline { 2 - 4 } & k2 and k5 & k2 and k6 & k3 and k6 \\
\hline \hline$N_{P C R}$ & 0.996049 & 0.996058 & 0.996009 \\
\hline$U_{A C I}$ & 0.334764 & 0.334787 & 0.334650 \\
\hline
\end{tabular}


Table 2. Stable of KM.

\begin{tabular}{|c|c|c|c|}
\hline & \multicolumn{3}{|c|}{ Fine Tuning KM } \\
\hline Cipher Text Image & Initial Key \& Fine Tuning k3 & Initial Key \& Fine Tuning k7 & Initial key \& Fine Tuning k3 and k7 \\
\hline \hline$N_{P C R}$ & 0.996049 & 0.996058 & 0.996009 \\
\hline$U_{A C I}$ & 0.334764 & 0.334787 & 0.334650 \\
\hline
\end{tabular}

for implementing $\mathrm{KM}$ and also robustly clarifies the reality of high failure rates, havin contributed to two researching areas of OC and KM but suffers two limitations.

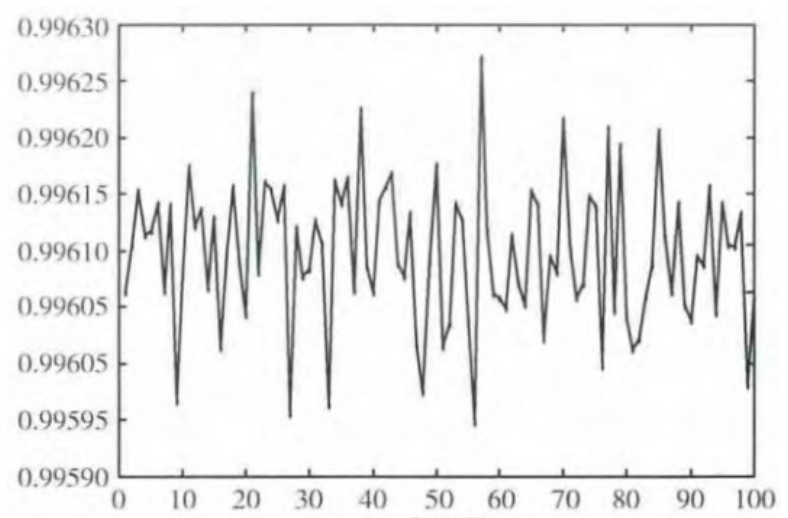

Fig. (1). The volatility of the sample.

The differential performance analysis is shown in Fig. (2), Among researches on the relationship between the two, most focus on the effects OC has on knowledge sharing or innovation, only a few getting involved in knowledge acquisition. In truth, $\mathrm{KM}$ is a comprehensive procedure containing multiple chains like identifying, gaining access to, integrating, spreading, sharing, innovating, and utilizing knowledge, and each chain acts as an indispensable determinant of successful KM operations.

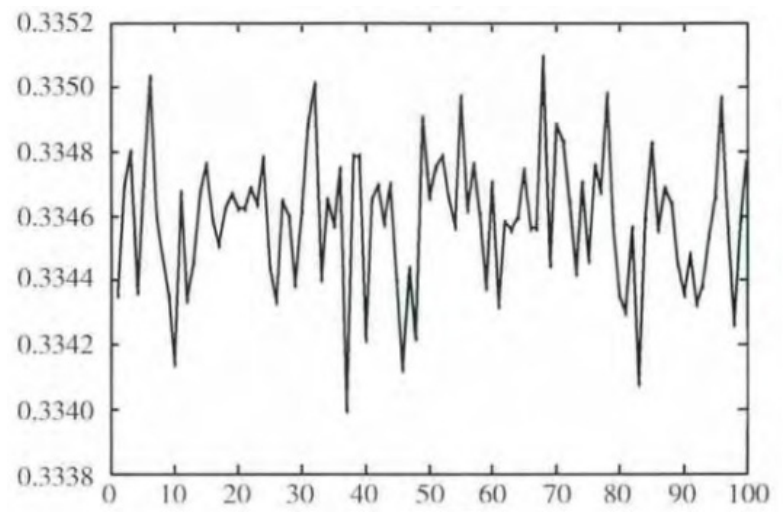

Fig. (2). Differential performance analysis.

\section{CONCLUSION}

OC exerts a profound effect on communications and behaviors during the $\mathrm{KM}$ course, a double-edged blade that provides a firm foundation for effective KM activities but might be a hurdle. This study probes into the effects of the three critical forms of cultural traits on KMS.
(1) Persistent learning, a basic feature in KMS operations and also essential requirements of KM, boosts the efficiency in gaining access to, accumulating, innovating, sharing and spreading knowledge, especially, its impact on knowledge acquisition is the most critical. (2) Interpersonal trust directly acts on the three links, that is, knowledge diffusion, knowledge innovation and knowledge application, to increasing the probability of KMS. One of the most notable is that interpersonal trust will decide directly the motivation of knowledge sharing and improve the efficiency of knowledge sharing; (3) A modest power distance is a contributor of improvement regarding the three aspects, with the most critical being in the impact on innovating knowledge.

Therefore, when the enterprise will push a program concerning KM for seeking KMS, it should be good at with creating the appropriate culture through suitable management system and action plan. The culture must be helpful of KM for the organization, at least in the continuous learning, interpersonal trust and power distance to the appropriate level. Meanwhile, related factors like support form high-level executives, investment in the scholarship administration infrastructure, and employee idiosyncrasy should be meditated as well. Ultimately, we expect that further empirical research on conciliating of these elements can yield a big and ripe fruit of KM activities.

\section{CONFLICT OF INTEREST}

The authors confirm that this article content has no conflict of interest.

\section{ACKNOWLEDGEMENTS}

Declared none.

\section{REFERENCES}

[1] S. Y. Chou, and Y. H. Chang, "A decision support system for supplier selection based on a strategy-aligned fuzzy SMART approach," Expert System with Applications, vol. 34, pp. 2241-2253, 2008.

[2] M. Dagdeviren, and I. Yuksel, "Developing a fuzzy analytic hierarchy process (AHP) model for behavior-based safety management," Information Sciences, vol. 178, no. 6, pp. 1717-1733, 2008.

[3] X.N. Fu, S.M. Yin and S.Q. Liu, "A improved adaptive fuzzy entropy entropy thresholding method on image segmentation", Acta Photonica Sinica, vol. 32, pp. 605-607, 2003.

[4] S. Gattoufi, M. Oral, and A. Reisman, "Data envelopment analysis literature: a bibliography update (1951-2001)," Socio-Economic Planning Sciences, no. 38, pp. 159-229, 2004.

[5] M. Keen, and J. Mintz, "The optimal threshold for a value-added tax," Journal of Public Economics, vol. 88, pp. 559-576, 2004.

[6] A. H. Gold, A. Malhotra, and A.H. Segars, "Knowledge management: an organizational capabilities perspective," Journal of Management Information Systems, vol. 11, pp. 185-217, 2001. 
[7] R.M. Grant, "Toward a knowledge-based theory of the firm," Strategic Management Journal, vol. 1, pp. 109-122, 1996.
[8] A.K. Gupta, and V. Govindarajan, "Knowledge flows within multinational corporations," Strategic Management Journal. vol. 21, pp. 473-496, 2000.

Received: June 10,2015

Revised: July 29, 2015

Accepted: August 15, 2015

(C) Qipeng et al.; Licensee Bentham Open.

This is an open access article licensed under the terms of the (https://creativecommons.org/licenses/by/4.0/legalcode), which permits unrestricted, noncommercial use, distribution and reproduction in any medium, provided the work is properly cited. 